\title{
Supporting Information: Quantifying Fluorogenic Dye Hydration in an Epoxy Resin by Non-Contact Microwave Dielectric Spectroscopy
}

\author{
Sindhu Seethamraju ${ }^{1,2}$, Jan Obrzut $^{1 *}$,Jack F. Douglas ${ }^{1 *}$, \\ Jeremiah W. Woodcock ${ }^{1}$ and Jeffrey W. Gilman ${ }^{1}$ \\ ${ }^{1}$ National Institute of Standards and Technology, Materials Science and Engineering Division \\ Gaithersburg MD, 20899-8542, USA \\ ${ }^{2}$ Department of Chemistry, Georgetown University, Washington, D.C., 20057-1227, USA
}

*Corresponding authors: jan.obrzut@nist.gov, jack.douglas@nist.gov

\section{Materials}

Rhodamine B, ethylene-di-amine, di-glicyl-ether (bis-phenol A DER 332, DGEBA) were obtained from Sigma Aldrich Ltd $^{\mathrm{a}}$. DGEBA was washed thoroughly with $\mathrm{NaOH}(1 \mathrm{~mol} / \mathrm{l})$ and water to remove the residual protons. Poly-ether-di-amine, (Jeffamine HK511 curing agent-amine) was purchased from Huntsman. Solvents such as chloroform, di-methylsulfoxide (DMSO), ethanol, ethyl-acetate, di-chloromethane, etc., of $99.9 \%$ purity were used in synthesis. Our water sensing fluorogenic dye, (AqFD was synthesized by reacting Rhodamine B $(0.2 \mathrm{~g}, 0.4 \mathrm{mmol})$, present in open form was reacted with ethylene-di-amine $(1.3 \mathrm{~g}, 21.6 \mathrm{mmol})$ in $10 \mathrm{~mL}$ of ethanol at $90{ }^{\circ} \mathrm{C}$, under reflux at $10{ }^{\circ} \mathrm{C}$ for $12 \mathrm{~h}$. The product, an open form of a spirolactam, is extracted using chloroform. It was further purified by column chromatography using ethyl acetate/hexanes 50/50 volume fraction. The samples were then dried under vacuum prior to their analysis by Nuclear Magnetic Resonance measurements.

${ }^{a}$ Certain commercial equipment, instruments, or materials are identified in this paper to foster understanding. Such identification does not imply recommendation or endorsement by the National Institute of Standards and Technology, nor does it imply that the materials or equipment identified are necessarily the best available for the purpose. 
AqFD was reacted with stochiometric amount of DGEBA. The modified AqFD-DGEBA was added to the curing mix of DGEBA with Jeffamine HK511 according to manufacturer specification. Samples were cured at $80{ }^{\circ} \mathrm{C}$ for $10 \mathrm{~h}$ under vacuum in metallic molds to obtain specimens with dimensions of $14 \mathrm{~mm} \times 8 \mathrm{~mm} \times 0.8 \mathrm{~mm}$. All the samples were prepared from the same master batch and at the same time. Controls without AqFD in the epoxy were also prepared for comparison.

Dry samples were baked in a vacuum oven for $12 \mathrm{~h}$ at $80{ }^{\circ} \mathrm{C}$ and stored under vacuum in the dark.

Water absorption was carried out from liquid phase by immersing dry specimens in deionized water under dark conditions, until desired concentration of absorbed water was obtained. Water uptake was determined gravimetrically by weighing using a microbalance. The wet samples were photo activated by exposing the samples to a $30 \mathrm{~W}$ UV light source for $3 \mathrm{~min}$.

\section{Methods:}

\section{FTIR Spectra}

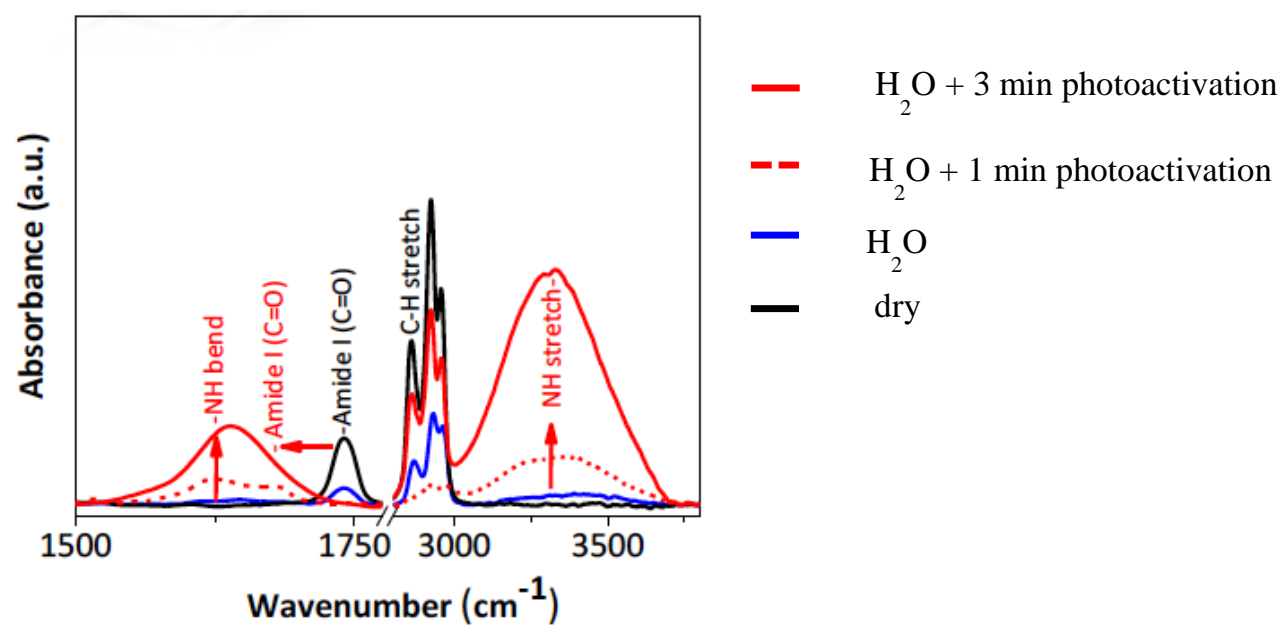

Figure S1. FTIR spectra of AqFD before and after activation 
The Fourier transform Infrared (FTIR) spectroscopy vibrational signatures for the carbonyl groups $(\mathrm{C}=\mathrm{O})$ are different when the $\gamma$-lactam is in closed and open forms.

Figure S1 shows that after photo activation (514 nm photons) and exposure to water, the peak for the amide-I $(\mathrm{C}=\mathrm{O})$ band of spirolactam $\left(1736 \mathrm{~cm}^{-1}\right)$ shifts towards $1683 \mathrm{~cm}^{-1}$ due to formation of the secondary amide. In addition, ring opening leads to the formation of a secondary amide bond. The absorption peaks in regions of $\approx 3200 \mathrm{~cm}^{-1}$ upon activation indicate the formation of $-\mathrm{NH}$, which is consistent with the ring opening mechanism. Heating above $80^{\circ} \mathrm{C}$ converts the AQ back to the closed spirolactam form.

\section{$\underline{\text { Dielectric Measurements }}$}

The complex relative permittivity, $\varepsilon$, of the epoxy specimen is the sum of real and imaginary parts $\left(\varepsilon=\varepsilon^{\prime}-j \varepsilon^{\prime \prime}\right)$ and is measured using a non-contact cavity perturbation method. This method of precise dielectric measurement is nondestructive and is experimentally simple to implement since it does not require any electrical contacts that could influenced by water adsorption.

In our earlier work, ${ }^{19}$ we showed that for a small specimen inside a rectangular cavity operating in the $\mathrm{TE}_{10}$ mode, the classical perturbation equation can be simplified to linear equations:

$$
\begin{gathered}
\mathrm{y}^{\prime}=\left(\varepsilon^{\prime}-1\right) 2 x-b^{\prime} \\
\mathrm{y}^{\prime \prime}=\varepsilon^{\prime \prime} 4 x-2 b^{\prime \prime}
\end{gathered}
$$

where $x, y^{\prime}$ and $y^{\prime \prime}$ are defined by the following relations:

$$
x=V_{s} / V_{0}, y^{\prime}=\left(f_{0} / f_{s}\right) / f_{0}, y^{\prime \prime}=1 / Q_{s}-1 / Q_{0}, b^{\prime} \text { and } b^{\prime \prime} \text { are constants }
$$


Here, $f_{0}$ is the resonant frequency of the empty cavity, $f_{\mathrm{s}}$ is the resonant frequency with the specimen present, $V_{0}$ is the volume of the cavity, $V_{\mathrm{s}}$ is the volume of the specimen $\left(V_{0} \gg V_{\mathrm{s}}\right) . Q_{0}$ is the resonance quality factor of the empty cavity and $Q_{\mathrm{s}}$ is the resonance quality factor of the cavity loaded with the specimen. The resonance quality factor is obtained from the resonant peak according to the conventional half power bandwidth formula as $Q_{\mathrm{s}}=f_{\mathrm{s}} / \mathrm{w} f_{\mathrm{s}}$. where $\mathrm{w} f_{\mathrm{s}}$ is the bandwidth of the resonant peak.

The real permittivity $\varepsilon_{\mathrm{r}}^{\prime}$ and the dielectric loss $\varepsilon_{\mathrm{r}}^{\prime \prime}$ can be determined from the slope of equations (1.a) and (1.b) respectively, where intercepts are the constants, $b^{\prime}$ and $b^{\prime \prime}$. Our cavity test fixture employs a WR90 waveguide, operating in the microwave frequency range of $6.7 \mathrm{GHz}$ to $13 \mathrm{GHz}$. The fixture is connected to a network analyzer (Agilent N5225A) with semi-rigid coaxial cables and near cross-polarized coaxial to WR90 coupling adapters. The network analyzer measures the transmission scattering parameter $\mathbf{S}_{21}$. The resonant frequency, $f_{\mathrm{s}}$, and the half power bandwidth, $\mathrm{w} f_{\mathrm{s}}$, are determined for the $\mathrm{TE}_{103}$ resonant mode. In this measurement we operate the cavity having $V_{0}=29.49672 \mathrm{~cm}^{3}$ at the resonant frequency of $7.4355 \mathrm{GHz}$, which corresponds to the $\mathrm{TE}_{103}$ resonant mode. The specimen is inserted into the cavity through a slot in the center of the cavity, where the $\mathrm{TE}_{103}$ electric field attains a maximum value. The specimen insertion and the corresponding volume of the material in the cavity $\left(V_{\mathrm{s}}\right)$ are controlled by a stage. During the measurements, the specimen is partially inserted in small steps, $\Delta V_{s}$, while the magnitude of the scattering parameter, $\mathbf{S}_{21}$, is recorded. The measured frequency span is typically $2 \mathrm{w} f_{s}$, recorded with a resolution of $10 \mathrm{kHz}$. The dynamic range of the noise level in the $\left|\mathbf{S}_{21}\right|$ magnitude is typically below $-65 \mathrm{~dB}$. The dielectric constant, $\varepsilon^{\prime}$, is determined with the combined uncertainty of $\pm 2 \times 10^{-3}$ and in 
the dielectric loss, $\varepsilon^{\prime \prime}$, the combined uncertainty is within $\pm 5 \times 10^{-4}$. The measurement data for eq $1 b$ are shown in Figure S2, where the slope of the plots equals to $\varepsilon^{\prime \prime}$.

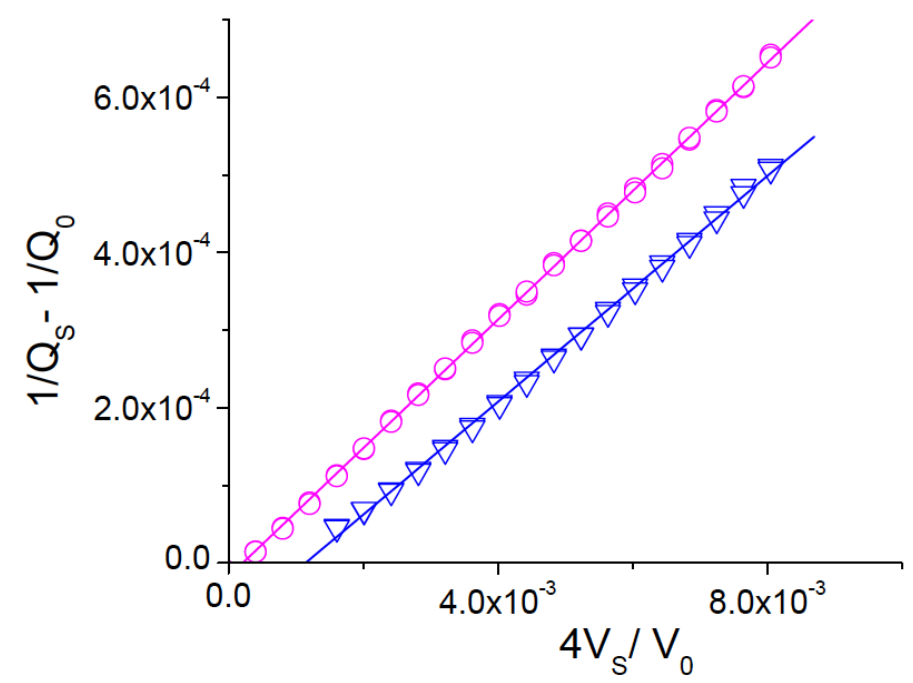

Figure S2. Plots of equation $1 \mathrm{~b}$ for $\varepsilon^{\prime \prime}$ having a total water content of $1 \%$ by mass; circles - epoxy with $1 \%$ AqFD by mass before activation and filled triangles-epoxy with $1 \%$ by mass AqFD after photo activation. Solid lines are linear fits through the data points.

From the slope of the plots in Fig S2 we find that before photo activation $\varepsilon^{\prime \prime}$ is 0.08254 $\pm 2.1 \times 10^{-4}$. After activation $\varepsilon_{r}^{\prime \prime}$ decreases to $0.0773 \pm 2.5 \times 10^{-4}$.

Uncertainty was estimated by applying the least squares fitting $\operatorname{model}^{1}$ to the dielectric loss data. The uncertainty in the dielectric measurement of $\varepsilon^{\prime \prime}$ illustrated in Fig S2 is typically within $2.5 \times 10^{-4}$. Uncertainty increases further from sample to sample after water uptake as shown in Fig. 3. The standard errors estimated from the leastsquares linear fitting of $\varepsilon^{\prime \prime}$ data points in Fig 3 are as follows: for epoxy $\left(2.4 \times 10^{-3}\right)$ epoxy- AqFD $\left(1.28 \times 10^{-3}\right)$ and epoxy-AqFD-activated $\left(3.53 \times 10^{-3}\right)$, which sum to standard uncertainty in $\Delta \varepsilon^{\prime \prime}$ to be about $6.4 \times 10^{-3}$. The combined standard uncertainty, 
$u$, of the complexation ratio, $\Delta \varepsilon^{\prime \prime} / \varepsilon^{\prime \prime}$, was then estimated from the square root of the variance model $^{28}$ :

$$
u\left(\Delta \varepsilon^{\prime \prime} / \varepsilon^{\prime \prime}\right)=\operatorname{SQRT}\left[1 /\left(\varepsilon^{\prime \prime}\right)^{2} u^{2}\left(\Delta \varepsilon^{\prime \prime}\right)+\left(\Delta \varepsilon^{\prime \prime}\right)^{2} /\left(\varepsilon^{\prime \prime}\right)^{4} u^{2}\left(\varepsilon^{\prime \prime}\right)\right]
$$

where $u\left(\Delta \varepsilon^{\prime \prime}\right)=6.4 \times 10^{-3}$ and $u\left(\varepsilon^{\prime \prime}\right)=2.5 \times 10^{-4}$.

The combined standard uncertainty of the complexation ratio obtained from Eq. (3), $u\left(\Delta \varepsilon^{\prime \prime} / \varepsilon^{\prime \prime}\right) \approx 2 \times 10^{-2}$, which is illustrated by the error bars in Figure 5. 